\title{
Supersonic relative velocity effect on the baryonic acoustic oscillation measurements
}

\author{
Yoo, J ; Dalal, N ; Seljak, U
}

\begin{abstract}
We investigate the effect of supersonic relative velocities between baryons and dark matter, recently shown to arise generically at high redshift, on baryonic acoustic oscillation (BAO) measurements at low redshift. The amplitude of the relative velocity effect at low redshift is model-dependent, but can be parameterized by using an unknown bias. We find that if unaccounted, the relative velocity effect can shift the BAO peak position and bias estimates of the dark energy equation-of-state due to its non-smooth, out-of-phase oscillation structure around the BAO scale. Fortunately, the relative velocity effect can be easily modeled in constraining cosmological parameters without substantially inflating the error budget. We also demonstrate that the presence of the relative velocity effect gives rise to a unique signature in the galaxy bispectrum, which can be utilized to isolate this effect. Future dark energy surveys can accurately measure the relative velocity effect and subtract it from the power spectrum analysis to constrain dark energy models with high precision.
\end{abstract}

DOI: https://doi.org/10.1088/1475-7516/2011/07/018

Posted at the Zurich Open Repository and Archive, University of Zurich

ZORA URL: https://doi.org/10.5167/uzh-54411

Journal Article

Accepted Version

Originally published at:

Yoo, J; Dalal, N; Seljak, U (2011). Supersonic relative velocity effect on the baryonic acoustic oscillation measurements. Journal of Cosmology and Astroparticle Physics, (7):18.

DOI: https://doi.org/10.1088/1475-7516/2011/07/018 


\title{
Supersonic relative velocity effect on the baryonic acoustic oscillation measurements
}

\author{
Jaiyul Yoo ${ }^{a, b}$ Neal Dalal $^{c}$ Uroš Seljak $^{a, b, d, e}$ \\ ${ }^{a}$ Institute for Theoretical Physics, University of Zürich, CH-8057 Zürich, Switzerland \\ ${ }^{b}$ Lawrence Berkeley National Laboratory, University of California, Berkeley, CA 94720, USA \\ ${ }^{c}$ Canadian Institute for Theoretical Astrophysics, University of Toronto, 60 St. George Street, Ontario, M5S \\ 3H8, Canada \\ ${ }^{d}$ Physics Department and Astronomy Department, University of California, Berkeley, CA 94720, USA \\ ${ }^{e}$ Institute for the Early Universe, Ewha Womans University, 120-750 Seoul, South Korea \\ E-mail: jyoo@physik.uzh.ch
}

\begin{abstract}
We investigate the effect of supersonic relative velocities between baryons and dark matter, recently shown to arise generically at high redshift, on baryonic acoustic oscillation (BAO) measurements at low redshift. The amplitude of the relative velocity effect at low redshift is model-dependent, but can be parameterized by using an unknown bias. We find that if unaccounted, the relative velocity effect can shift the BAO peak position and bias estimates of the dark energy equation-of-state due to its non-smooth, out-of-phase oscillation structure around the BAO scale. Fortunately, the relative velocity effect can be easily modeled in constraining cosmological parameters without substantially inflating the error budget. We also demonstrate that the presence of the relative velocity effect gives rise to a unique signature in the galaxy bispectrum, which can be utilized to isolate this effect. Future dark energy surveys can accurately measure the relative velocity effect and subtract it from the power spectrum analysis to constrain dark energy models with high precision.
\end{abstract}

Keywords: large scale structure of the Universe, baryonic acoustic oscillations, power spectrum 


\section{Contents}

1 Introduction $\quad 1$

2 Supersonic relative velocity effect $\quad 2$

3 Separating relative velocity contribution: Bispectrum $\quad 4$

4 Effect on BAO measurements: Power spectrum $\quad 7$

4.1 Relative velocity contribution and galaxy power spectrum $\quad 7$

$\begin{array}{ll}\text { 4.2 Shift in the BAO peak position } & 9\end{array}$

5 Cosmological constraining power and bias in parameter estimation 10

6 Discussion $r$

$\begin{array}{lr}\text { A Power spectrum and bispectrum computation } & 14\end{array}$

B Parameter forecast and bias in the parameter estimation $\quad 15$

\section{Introduction}

Understanding the nature of dark energy is one of the key questions in contemporary science, and various techniques have been developed over the past decade to advance this goal. Measurements of the baryonic acoustic oscillation (BAO) feature in galaxy surveys provide an especially promising way to constrain the expansion history of the universe and the behavior of dark energy [1]. The physical scale of the acoustic oscillation is determined with high accuracy by cosmic microwave background (CMB) measurements (e.g., $[2,3])$, making BAO measurements at low redshift a robust standard ruler. Since current and future dark energy surveys aim to measure the BAO peak position at sub-percent level precision, significant efforts have been devoted to modeling nonlinear evolution and its effect on the BAO peak position [4-11]. At low redshift, where nonlinear effects are substantial on BAO scales, the BAO peak contrast is degraded and the peak position may be subtly shifted compared to the linear theory prediction. However, these nonlinear effects tend to produce smooth, broad-band power and may readily be removed by template fitting or explicit modeling of these effects [12-14].

Recently, Tseliakhovich and Hirata [15] discussed a new nonlinear effect in the growth of small scale structure at very early times. Prior to the recombination epoch, baryons are tightly coupled to the photons, while dark matter is decoupled from the baryon-photon fluid and its fluctuation grows logarithmically with small velocity. After cosmic recombination, when the tight coupling of the baryon-photon fluid is broken and the gas sound speed plummets, the relative velocity between the baryon and the dark matter fluids becomes supersonic. These random relative velocities effectively increase the Jeans mass for the formation of the earliest baryonic structure, thereby suppressing their abundance [15-17]. High-resolution numerical simulations [18, 19] have confirmed that supersonic relative velocities raise the minimum halo mass that can form the first stars, and delay star formation in time, by an amount that depends on the magnitude of the relative velocity (see also [20]). This modulation of baryonic structures at high redshift imprints signatures of the relative velocity effect in the power spectrum of objects such as minihalos at high redshift $[15,16]$, and hence the power spectrum of any observable which traces these objects can exhibit significant departures from simple linear biasing of the matter power spectrum on $\mathrm{BAO}\left(\sim 100 h^{-1} \mathrm{Mpc}\right)$ scales.

Even at low redshift $z \lesssim 5$, when massive dark matter halos form, the relative velocity effect can be indirectly important, contrary to the naive expectation that this effect becomes negligible within halos whose velocity dispersion greatly exceeds the relative velocity. One possible scenario [16] is that patchy reionization, driven by early minihalos, inherits the imprints of the relative velocity effect in the spatial distribution of early minihalos, resulting in a spatial variation of the subsequent star formation history modulated by the large-scale 
power of the relative velocity effect. Consequently, massive galaxies at low redshift may retain the memory of the large-scale relative velocity effect seen in the minihalos at high redshift. Even tiny $\sim 1 \%$ level effects on the colors or luminosities of massive galaxies can have significant implications, since these effects will be correlated across $\sim 100 h^{-1} \mathrm{Mpc}$ length scales. Any residual relative velocity effect in low-redshift massive galaxies may have significant impact on the precision measurements of the BAO peak position. Since the power spectrum of relative velocities has a prominent oscillation structure that is out-of-phase with the matter power spectrum, it cannot be removed by a blind broad-band fitting of the galaxy power spectrum, and therefore it can potentially bias the determination of the BAO peak position.

The primary purpose of the present work is to investigate the impact of any relative velocity effect that persists in low-redshift massive galaxies, on our ability to probe the underlying cosmology using galaxy power spectrum measurements, and to find possible ways to isolate and remove this effect in the analysis. The organization of this paper is as follows. In section 2 we briefly describe the supersonic relative velocity effect and its impact on the earliest baryonic structure. In section 3 we model the observed galaxy as a tracer of the underlying matter and the relative velocity distributions and compute the galaxy bispectrum to isolate the relative velocity contribution. We then investigate the impact of the relative velocity effect on the BAO measurements in section 4 . We first compute the full power spectrum including the relative velocity effect in section 4.1 and quantify the shift in the BAO peak position due to the relative velocity effect in section 4.2. In section 5 we perform a Fisher matrix analysis to investigate the impact of the relative velocity effect on the cosmological parameter estimation and how well the relative velocity effect can be measured by using the bispectrum. Finally, we conclude in section 6 with a discussion of further implication. Technical details of our derivations are presented in Appendices A and B.

Here we present our calculations assuming a flat $\Lambda$ CDM universe with the matter density $\omega_{m}=\Omega_{m} h^{2}=$ $0.134\left(\Omega_{m}=0.271\right)$, the baryon density $\omega_{b}=\Omega_{b} h^{2}=0.0222\left(\Omega_{b}=0.045\right)$, the spectral index $n_{s}=0.966$ and its running $\alpha_{s}=0$ of the primordial curvature power spectrum $\Delta_{\varphi}^{2}=2.42 \times 10^{-9}\left(\sigma_{8}=0.81\right)$ at $k_{0}=0.002 \mathrm{Mpc}^{-1}$ (consistent with the Wilkinson Microwave Anisotropy Probe measurements [2, 3] and the Sloan power spectrum measurements [21, 22]).

\section{Supersonic relative velocity effect}

Before the recombination epoch, baryons and photons are tightly coupled with sound speed $c_{s} \simeq 1 / \sqrt{3}$, while dark matter is decoupled and cold. At the release of baryons following cosmic recombination, the gas sound speed drops precipitously $\left(c_{s} \sim 10^{-5}\right)$, rendering the baryon fluids supersonic $(\mathcal{M} \simeq 2-5)$. The different velocities of the dark matter and the baryon fluids result in suppression of the matter power spectrum at the Jeans scale $\left(k_{\mathrm{J}} \sim 200 \mathrm{Mpc}^{-1}\right)$ and suppression of the growth of structure at the relative velocity scale $\left(k_{r}=k_{\mathrm{J}} / \mathcal{M}, M_{\mathrm{h}} \simeq 10^{6} M_{\odot} \sim k_{r}^{-3}\right)[15,17]$. Furthermore, the relative velocity effect modulated by largescale acoustic oscillations imprints its signature in the collapsed baryon fraction at early time, and this effect can be used to probe the nature of minihalos before reionization [16].

The velocities $\mathbf{v}_{b, m}$ of the baryon and dark matter distributions are related to their density fluctuations as

$$
\mathbf{v}_{b, m}(\mathbf{k}, z)=i \frac{\mathbf{k}}{k^{2}} a \dot{\delta}_{b, m}(\mathbf{k}, z)=i \frac{\mathbf{k}}{k^{2}} a \dot{T}_{b, m}(k, z) \varphi_{p}(\mathbf{k})
$$

where $\varphi_{p}(\mathbf{k})$ is the primordial curvature perturbation in the total comoving gauge. The evolved density fluctuations are computed using the Einstein-Boltzmann code CMBFAST [23] and are expressed in terms of their transfer functions $\delta_{b, m}(\mathbf{k}, z)=T_{b, m}(k, z) \varphi_{p}(\mathbf{k})$. Instead of taking the derivatives of the transfer functions, we simply use the velocity transfer function $T_{v}(k, z)$ from the CMBFAST code (it is related to the density transfer function as $\left.T_{v b, v m}=-a \dot{T}_{b, m} / k\right)$. The relative velocity of the baryon and the dark matter fluids is then $\mathbf{v}_{r}=\mathbf{v}_{b}-\mathbf{v}_{m}$, and we define the dimensionless relative velocity as $\mathbf{u}_{r}=\mathbf{v}_{r} / \sigma_{r v}$ (and their transfer function $T_{r u}$ as well) using the one-dimensional rms relative velocity fluctuation $\sigma_{r v}$. Their statistical properties are completely determined by the two-point correlation function

$$
\psi_{i j}(r)=\left\langle u_{r}^{i}(\mathbf{x}) u_{r}^{j}(\mathbf{x}+\mathbf{r})\right\rangle=\psi_{\perp}(r) \delta_{i j}+\left[\psi_{\|}(r)-\psi_{\perp}(r)\right] \delta_{i z} \delta_{j z}=\int \frac{d^{3} \mathbf{k}}{(2 \pi)^{3}} e^{i \mathbf{k} \cdot \mathbf{r}} P_{u}^{i j}(\mathbf{k})
$$



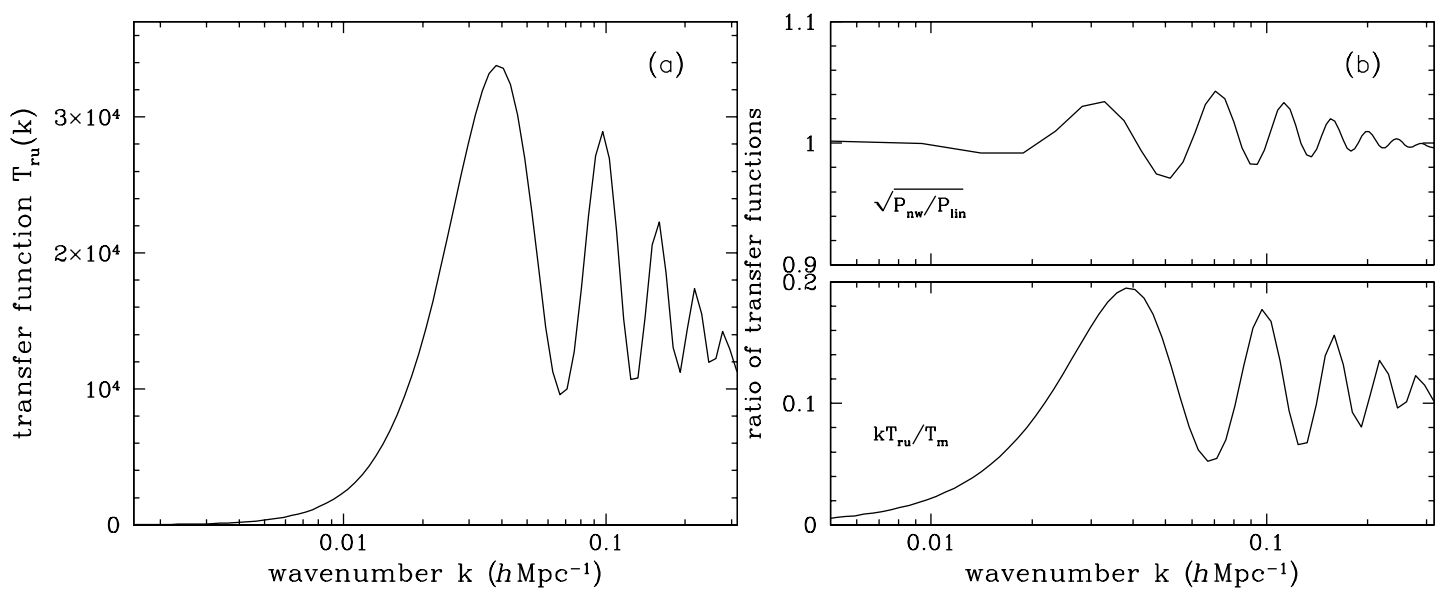

Figure 1. Transfer functions and correlation functions. (a) Relative velocity transfer function $T_{r u}(k)$ at $z=0$. (b) Ratio of the relative velocity to the dark matter transfer functions $k T_{r u} / T_{m}$ (bottom panel). For comparison, the upper panel shows the ratio of the no-wiggle power spectrum to the linear matter power spectrum. The transfer functions are defined with respect to the primordial curvature perturbation, and the no-wiggle power spectrum is computed by using the Eisenstein and $\mathrm{Hu}[24]$ fit to the smooth power spectrum shape.

where the indices $i, j$ represent the spatial component of the relative velocity vector, the $z$-direction is set parallel to the separation vector $\mathbf{r}\left(\psi_{\perp}=\psi_{x x}=\psi_{y y}, \psi_{\|}=\psi_{z z}\right)$, and the relative velocity power spectrum is defined as

$$
\left\langle u_{r}^{i}\left(\mathbf{k}_{a}\right) u_{r}^{j}\left(\mathbf{k}_{b}\right)\right\rangle=(2 \pi)^{3} \delta^{D}\left(\mathbf{k}_{a}+\mathbf{k}_{b}\right) P_{u}^{i j}\left(\mathbf{k}_{a}\right)
$$

with

$$
P_{u}^{i j}(\mathbf{k})=\frac{k^{i} k^{j}}{k^{4}}\left[\frac{a \dot{T}_{r}(k)}{\sigma_{r v}}\right]^{2} P_{\varphi}(k)=\frac{k^{i} k^{j}}{k^{2}} T_{r u}^{2}(k) P_{\varphi}(k)=\frac{k^{i} k^{j}}{k^{2}} \frac{T_{r u}^{2}(k)}{T_{m}^{2}(k)} P_{m}(k) .
$$

For notational simplicity, we suppressed the time dependence of the transfer functions and their power spectra.

Figure 1a shows the transfer function $T_{r u}(k)$ of the relative velocity effect at $z=0$, where its amplitude indicates the linear growth of power given the primordial curvature power spectrum $\Delta_{\varphi}^{2}(k)=A\left(k / k_{0}\right)^{n_{s}-1}$ with $A=2.4 \times 10^{-9}$ and $k_{0}=0.002 \mathrm{Mpc}^{-1}$. The relative velocity is predominantly sourced by structures on size scales of order the acoustic scale; larger-scale structures make little contribution, and the transfer function $T_{r u}(k)$ declines rapidly at $k \lesssim 0.03 \mathrm{~h} \mathrm{Mpc}^{-1}$ since baryons and dark matter behave similarly on scales beyond the sound horizon at decoupling. The ratio of the relative velocity to the dark matter transfer functions $T_{r u}(k) / T_{m}(k)$ is shown in Fig. $1 \mathrm{~b}$, where the scaling factor $1 / k$ reflecting energy-momentum conservation is removed. The prominent oscillation structure displayed in Fig. 1a is still visible in the bottom panel of Fig. 1b, when compared to the matter transfer function. To facilitate the comparison of the acoustic oscillation amplitude and its phase in the relative velocity and the dark matter transfer functions, we compute the ratio of the no-wiggle power spectrum to the linear matter power spectrum and plot its square root in the upper panel of Fig. 1b, where the no-wiggle power spectrum is a fit to the smooth shape of the linear matter power spectrum [24]. The oscillation structure in the relative velocity and the matter distributions is out-of-phase, and its amplitude $(\sim 5 \%)$ is smaller in the matter distribution as the acoustic oscillation was only present in the baryon distribution, not in the dark matter distribution at early time. By contrast, the relative velocity arises entirely from the acoustic oscillations, and hence its oscillation amplitude is fractionally order one.

However, the relative velocity of the baryon and the dark matter distributions is not directly observable. Dalal et al. [16] argue that the collapsed baryon fraction at early time is affected by the relative velocity effect as it changes the effective sound speed and increases the equivalent Jeans mass for baryonic gas to collapse in 
dark matter halos. The two-point correlation function of the collapsed baryon fraction can be computed [16] as

$$
\xi_{f}(r)=b_{r}^{2}\left[\psi_{\perp}^{2}(r)+\frac{1}{2} \psi_{\|}^{2}(r)\right] \propto\left\langle u_{r}^{2}(\mathbf{x}) u_{r}^{2}(\mathbf{x}+\mathbf{r})\right\rangle
$$

implying that the collapsed baryon fraction is a biased tracer of the relative velocity $u_{r}^{2}(\mathbf{x})$. We quantify the effect of the relative velocity on the BAO peak shift in section 4.

\section{Separating relative velocity contribution: Bispectrum}

At high redshift, $z>10$, the supersonic relative velocity allows baryons to advect out of small dark matter halos, effectively increasing the Jeans mass of the gas in a velocity-dependent manner, thereby modulating the large-scale clustering of collapsed baryonic objects [16]. Additionally, the abundance of dark matter halos is also modulated by a similar effect [15]. Quite generally, we can write the large-scale fluctuation of the collapsed objects as [16]

$$
\delta_{g}(\mathbf{x})=b_{1} \delta_{m}(\mathbf{x})+b_{r}\left[u_{r}^{2}(\mathbf{x})-\sigma_{r u}^{2}\right],
$$

where $b_{1}$ and $b_{r}$ are the bias parameters of the collapsed halo with respect to the matter density and the relative velocity, respectively. Hereafter, we collectively call the collapsed baryon and dark matter system a galaxy, though a galaxy is often used to refer to a baryon only system and at high redshift the collapsed baryon and dark matter systems may appear different from typical "galaxies" at low redshift. The galaxy fluctuation is not only a tracer of the underlying matter distribution, but also a tracer of the relative velocity. The magnitude of the relative velocity bias $b_{r}$ is computed by Dalal et al. [16] at $z>10$, where the collapse of the baryonic structure can be relatively simply modeled. As discussed above, the relative velocity between baryons and dark matter can indirectly modulate the properties of galaxies at low redshift. The large-scale clustering properties of observed galaxies can, to lowest order, therefore be written in the form of eq. (3.1). In this case the velocity bias $b_{r}$ is treated as a free parameter, reflecting our great uncertainty in the physical processes that determine galaxy properties at low redshift.

Since the relative velocity $u_{r}$ enters quadratically in eq. (3.1), it is readily apparent that the relative velocity effect will lead to a nonvanishing bispectrum of the galaxy fluctuation, providing a direct way to isolate its contribution from the matter density distribution. Noting that the relative velocity has no directional dependence, the ensemble average of the galaxy fluctuations can be computed as

$$
\begin{aligned}
& \left\langle\delta_{g}\left(\mathbf{k}_{a}\right) \delta_{g}\left(\mathbf{k}_{b}\right) \delta_{g}\left(\mathbf{k}_{c}\right)\right\rangle=b_{1}^{2} b_{r} \int \frac{d^{3} \mathbf{q}}{(2 \pi)^{3}} \sum_{i=x, y, z}\left\langle\delta_{g}\left(\mathbf{k}_{a}\right) \delta_{g}\left(\mathbf{k}_{b}\right) u_{r}^{i}(\mathbf{q}) u_{r}^{i}\left(\mathbf{k}_{c}-\mathbf{q}\right)\right\rangle+\text { cyclic permutations } \\
& \quad+b_{r}^{3} \int \frac{d^{3} \mathbf{q}_{1}}{(2 \pi)^{3}} \int \frac{d^{3} \mathbf{q}_{2}}{(2 \pi)^{3}} \int \frac{d^{3} \mathbf{q}_{3}}{(2 \pi)^{3}} \sum_{i, j, k}\left\langle u_{r}^{i}\left(\mathbf{q}_{1}\right) u_{r}^{i}\left(\mathbf{k}_{a}-\mathbf{q}_{1}\right) u_{r}^{j}\left(\mathbf{q}_{2}\right) u_{r}^{j}\left(\mathbf{k}_{b}-\mathbf{q}_{2}\right) u_{r}^{k}\left(\mathbf{q}_{3}\right) u_{r}^{k}\left(\mathbf{k}_{c}-\mathbf{q}_{3}\right)\right\rangle
\end{aligned}
$$

Counting only the connected part $\left(\mathbf{k}_{a}+\mathbf{k}_{b}+\mathbf{k}_{c}=0\right)$ in eq. (3.2), we can derive the galaxy bispectrum (see Appendix A)

$$
\begin{aligned}
& B_{g}\left(\mathbf{k}_{a}, \mathbf{k}_{b}, \mathbf{k}_{c}\right)=2 b_{1}^{2} b_{r} \sum_{i=x, y, z}\left[P_{\times}^{i}\left(\mathbf{k}_{a}\right) P_{\times}^{i}\left(\mathbf{k}_{b}\right)+\operatorname{cycl} .\right]+8 b_{r}^{3} \int \frac{d^{3} \mathbf{q}}{(2 \pi)^{3}} \sum_{i, j, k} P_{u}^{i j}\left(\mathbf{k}_{a}+\mathbf{q}\right) P_{u}^{j k}\left(\mathbf{k}_{b}-\mathbf{q}\right) P_{u}^{k i}(\mathbf{q}) \\
& \quad=2 b_{1}^{2} b_{r}\left[P_{m}\left(k_{a}\right) P_{m}\left(k_{b}\right) G_{u}\left(\mathbf{k}_{a}, \mathbf{k}_{b}\right)+P_{m}\left(k_{b}\right) P_{m}\left(k_{c}\right) G_{u}\left(\mathbf{k}_{b}, \mathbf{k}_{c}\right)+P_{m}\left(k_{c}\right) P_{m}\left(k_{a}\right) G_{u}\left(\mathbf{k}_{c}, \mathbf{k}_{a}\right)\right] \\
& \quad+8 b_{r}^{3} \int \frac{d^{3} \mathbf{q}}{(2 \pi)^{3}} P_{m}\left(\left|\mathbf{k}_{a}+\mathbf{q}\right|\right) P_{m}\left(\left|\mathbf{k}_{b}-\mathbf{q}\right|\right) P_{m}(q) G_{u}\left(\mathbf{k}_{a}+\mathbf{q}, \mathbf{k}_{b}-\mathbf{q}\right) G_{u}\left(\mathbf{k}_{a}+\mathbf{q}, \mathbf{q}\right) G_{u}\left(\mathbf{q}, \mathbf{k}_{b}-\mathbf{q}\right)
\end{aligned}
$$

where the cross-power spectrum of the matter density and the relative velocity is defined by

$$
\left\langle\delta_{m}\left(\mathbf{k}_{a}\right) u_{r}^{i}\left(\mathbf{k}_{b}\right)\right\rangle=(2 \pi)^{3} \delta^{D}\left(\mathbf{k}_{a}+\mathbf{k}_{b}\right) P_{\times}^{i}\left(\mathbf{k}_{a}\right)=-\left\langle u_{r}^{i}\left(\mathbf{k}_{a}\right) \delta_{m}\left(\mathbf{k}_{b}\right)\right\rangle
$$

as

$$
P_{\times}^{i}(\mathbf{k})=-i \frac{k^{i}}{k^{2}} \frac{a \dot{T}_{r}(k)}{\sigma_{r v}} T_{m}(k) P_{\varphi}(k)=i \frac{k^{i}}{k} T_{r u}(k) T_{m}(k) P_{\varphi}(k)=i \frac{k^{i}}{k} \frac{T_{r u}(k)}{T_{m}(k)} P_{m}(k)
$$



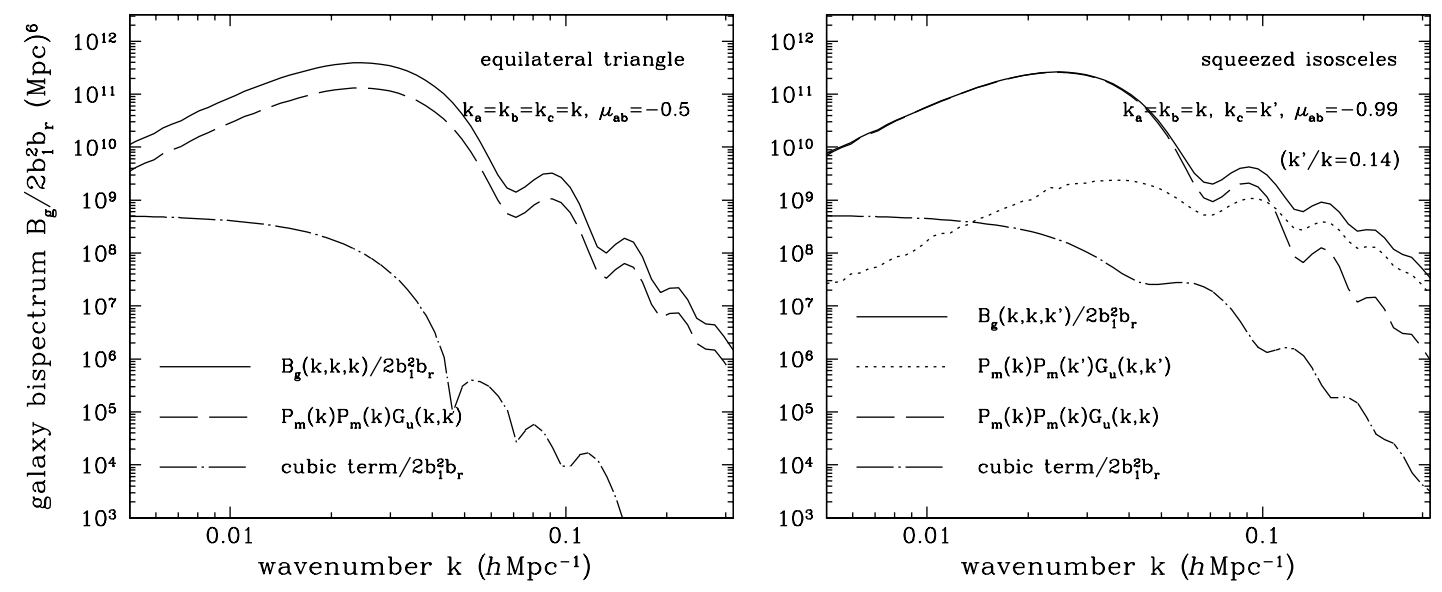

Figure 2. Relative velocity contributions to the galaxy bispectrum for an equilateral (left) and a squeezed isosceles (right) triangular shapes. Dotted, dashed, and dot-dashed lines show each component of the galaxy bispectrum in eq. (3.3), and their sum is shown as solid lines. The bias parameter $b_{r} / b_{1}=0.01$ is assumed for computing the cubic term in eq. (3.3).

and the relative velocity kernel is

$$
G_{u}\left(\mathbf{k}_{a}, \mathbf{k}_{b}\right)=-\frac{a^{2}}{\sigma_{r v}^{2}} \frac{\dot{T}_{v}\left(k_{a}\right)}{T_{m}\left(k_{a}\right)} \frac{\dot{T}_{v}\left(k_{b}\right)}{T_{m}\left(k_{b}\right)} \frac{\mathbf{k}_{a} \cdot \mathbf{k}_{b}}{k_{a}^{2} k_{b}^{2}}=-\frac{T_{r u}\left(k_{a}\right)}{T_{m}\left(k_{a}\right)} \frac{T_{r u}\left(k_{b}\right)}{T_{m}\left(k_{b}\right)} \mu_{a b},
$$

with $\mu_{a b}=\mathbf{k}_{a} \cdot \mathbf{k}_{b} / k_{a} k_{b}$.

Figure 2 illustrates the galaxy bispectrum for two triangular configurations. The left panel examines the scale-dependence of the galaxy bispectrum for an equilateral shape $\left(k_{a}=k_{b}=k_{c}=k, \mu=-0.5\right)$. With the relative velocity kernel $G_{u}(k, k) \propto k^{-2}$ on scales of interest, the galaxy bispectrum declines sharply on small scales in proportion to $k^{-6.5}$, while it flattens on large scales. However, because baryons and dark matter have similar velocities on large scales $k \lesssim 0.03 \mathrm{hMpc}^{-1}$ seen in Fig. 1a, this term in the galaxy bispectrum asymptotically vanishes. The acoustic oscillation structure of the galaxy bispectrum around $k \simeq 0.1 \mathrm{hMpc}^{-1}$ reflects the oscillation due to the relative velocity effect. Being a convolution, the cubic contribution (dotdashed) in eq. (3.3) is constant and nonvanishing on large scales, because the contribution to the cubic term arises at $q \simeq 0.01-0.1 \mathrm{hMpc}^{-1}$. The right panel illustrates the scale-dependence of the galaxy bispectrum for a squeezed isosceles triangle $\left(k_{a}=k_{b}=k \neq k_{c}, \mu_{a b}=-0.99\right)$. Since the third scale of the isosceles is larger $k_{c}=k \sqrt{2+2 \mu}=0.14 k$ and the scaling of the bispectrum is steep, two contributions $P_{m}(k) P_{m}\left(k_{c}\right) G_{u}\left(k, k_{c}\right)$ in eq. (3.3) that mix different scales $k$ and $k_{c}$ are larger than the other term $P_{m}(k) P_{m}(k) G_{u}(k, k)$ on small scales, but it falls over faster on large scales. The cubic term as in the equilateral configuration is subdominant for $b_{r} / b_{1} \leq 0.1$ at $k \geq 0.01 \mathrm{hpc}^{-1}$.

At low redshift, however, the matter fluctuation develops substantial nonlinearity on small scales and is quasi-linear even at relatively large scale $k \simeq 0.1 \mathrm{hMpc}^{-1}$, demanding treatment beyond linear order in eq. (3.1). The nonlinear evolution in the matter density distribution results in a nonvanishing bispectrum of the galaxy fluctuation even in the absence of the relative velocity effect. Here we adopt standard perturbation theory to compute the nonlinear terms in the matter density distribution to the third order (e.g., [25-27]): $\delta_{m}(\mathbf{k})=\delta_{m}^{(1)}(\mathbf{k})+\delta_{m}^{(2)}(\mathbf{k})+\delta_{m}^{(3)}(\mathbf{k})$, where the superscript indicates the perturbation order and the secondorder matter fluctuation is

$$
\delta_{m}^{(2)}(\mathbf{k})=\int \frac{d^{3} \mathbf{q}}{(2 \pi)^{3}} \delta_{m}^{(1)}(\mathbf{q}) \delta_{m}^{(1)}(\mathbf{k}-\mathbf{q}) F_{2}(\mathbf{q}, \mathbf{k}-\mathbf{q})
$$



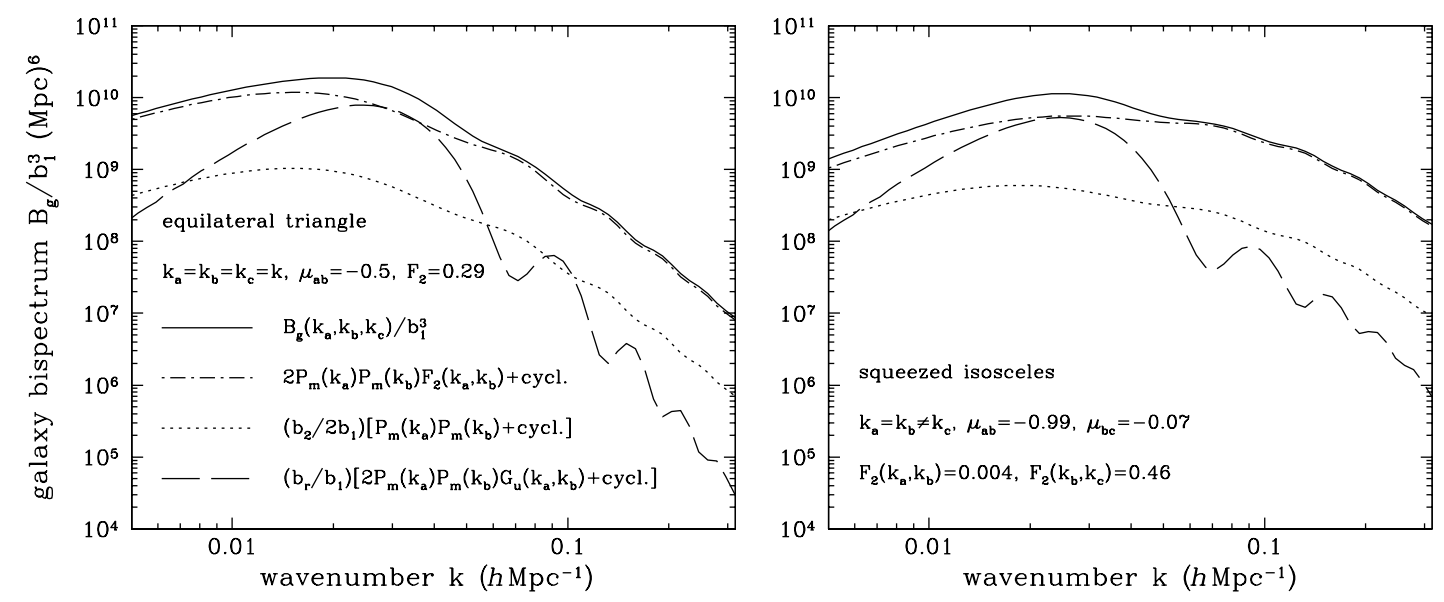

Figure 3. Scale-dependence of the full galaxy bispectrum for two triangular shapes. Three different components contribute to the full galaxy bispectrum in eq. (3.10): The nonlinear evolution of the matter density distribution (dot-dashed), the nonlinear bias (dotted), and the relative velocity effect (dashed). The cubic term in eq. (3.10) is omitted to avoid clutter. The full galaxy bispectrum is shown as solid lines. The bias parameters $b_{2} / b_{1}=0.1$ and $b_{r} / b_{1}=0.01$ are assumed.

with the second-order kernel

$$
F_{2}\left(\mathbf{k}_{a}, \mathbf{k}_{b}\right)=\frac{5}{7}+\frac{2}{7}\left(\frac{\mathbf{k}_{a} \cdot \mathbf{k}_{b}}{k_{a} k_{b}}\right)^{2}+\frac{\mathbf{k}_{a} \cdot \mathbf{k}_{b}}{2}\left(\frac{1}{k_{a}^{2}}+\frac{1}{k_{b}^{2}}\right) .
$$

Along with the nonlinear gravitational evolution in the matter density distribution, we also need to consider nonlinear galaxy bias. Expanding to third order, we model the galaxy fluctuation as

$$
\delta_{g}(\mathbf{x})=b_{1} \delta_{m}(\mathbf{x})+\frac{b_{2}}{2}\left[\delta_{m}^{2}(\mathbf{x})-\sigma_{m}^{2}\right]+\frac{b_{3}}{3 !} \delta_{m}^{3}(\mathbf{x})+b_{r}\left[u_{r}^{2}(\mathbf{x})-\sigma_{r u}^{2}\right],
$$

where we keep only the linear order term in the relative velocity effect as it is the relic effect of the early universe and it decays with the expansion factor $a$, rendering higher-order terms in $u_{r}^{2}(\mathbf{x})$ negligible at low redshift. Therefore, the full bispectrum of the galaxy fluctuation in eq. (3.9) is

$$
\begin{aligned}
& B_{g}\left(\mathbf{k}_{a}, \mathbf{k}_{b}, \mathbf{k}_{c}\right)=b_{1}^{3}\left[2 P_{m}\left(k_{a}\right) P_{m}\left(k_{b}\right) F_{2}\left(\mathbf{k}_{a}, \mathbf{k}_{b}\right)+\mathrm{cycl} .\right]+\frac{1}{2} b_{1}^{2} b_{2}\left[P_{m}\left(k_{a}\right) P_{m}\left(k_{b}\right)+\text { cycl. }\right] \\
& +b_{1}^{2} b_{r}\left[2 P_{m}\left(k_{a}\right) P_{m}\left(k_{b}\right) G_{u}\left(\mathbf{k}_{a}, \mathbf{k}_{b}\right)+\mathrm{cycl} .\right] \\
& +8 b_{r}^{3} \int \frac{d^{3} \mathbf{q}}{(2 \pi)^{3}} P_{m}\left(\left|\mathbf{k}_{a}+\mathbf{q}\right|\right) P_{m}\left(\left|\mathbf{k}_{b}-\mathbf{q}\right|\right) P_{m}(q) G_{u}\left(\mathbf{k}_{a}+\mathbf{q}, \mathbf{k}_{b}-\mathbf{q}\right) G_{u}\left(\mathbf{k}_{a}+\mathbf{q}, \mathbf{q}\right) G_{u}\left(\mathbf{q}, \mathbf{k}_{b}-\mathbf{q}\right)
\end{aligned}
$$

where $P_{m}(k)$ is the linear matter power spectrum. In addition to the relative velocity contributions in eq. (3.3), the full galaxy bispectrum receives two more contributions from the nonlinear evolution: The first squarebracket represents the contributions of the nonlinear evolution in the matter density distribution, while the second square-bracket represents the nonlinear bias contributions described in eq. (3.9).

Figure 3 plots the scale-dependence of the full galaxy bispectrum in eq. (3.10). The left panel dissects each component of the galaxy bispectrum given an equilateral triangular shape $\left(k_{a}=k_{b}=k_{c}=k, \mu=-0.5\right.$, $\left.F_{2}=0.29\right)$. In this and subsequent figures, we assume the nonlinear bias parameter $b_{2} / b_{1}=0.1$ and the relative velocity bias parameter $b_{r} / b_{1}=0.01$ as our fiducial bias parameters for illustration. Two contributions from the nonlinear bias (dotted) and matter density (dot-dashed) are comparable in amplitude if $b_{2} \simeq b_{1}$, and they are dominant over the relative velocity effect (dashed) on small scales. However, the relative velocity 


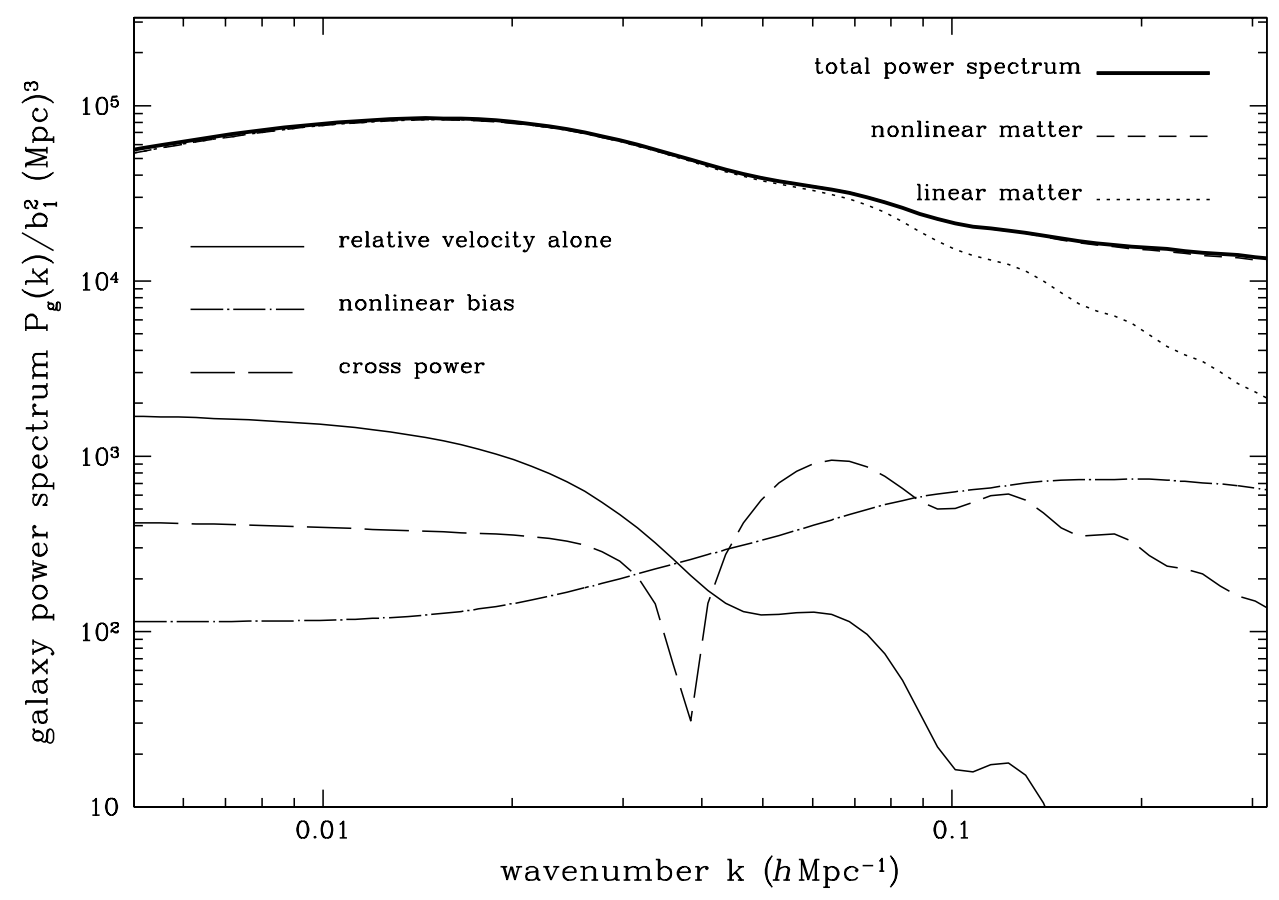

Figure 4. Full galaxy power spectrum with the bias parameters $b_{2} / b_{1}=0.1$ and $b_{r} / b_{1}=0.01$. The galaxy power spectrum (thick solid) is computed by using eq. (4.2). Thin solid, long dashed, and dot-dashed lines represent the autopower spectrum of the relative velocity effect $\left(\sim b_{r}^{2}\right)$, the cross-power spectrum of the relative velocity and the nonlinear galaxy bias $\left(\sim b_{r}\right)$, and the auto-power spectrum of the nonlinear galaxy bias, respectively. The latter (long dashed) becomes negative at $k=0.04 \mathrm{~h} \mathrm{Mpc}^{-1}$, beyond which its absolute value is plotted. The linear and the nonlinear matter power spectra are shown as dotted and short dashed lines (the short dashed line is largely obscured by the thick solid line).

contribution is comparable to the matter density contribution at large scale $k \simeq 0.03 \mathrm{hMpc}^{-1}$, corresponding to the peak seen in Fig. 1a. The right panel shows the galaxy bispectrum for a squeezed isosceles shape $\left(k_{a}=k_{b}=k \neq k_{c}, \mu_{a b}=-0.99\right)$. For the squeezed configuration, the large scale power $P_{m}\left(k_{c}\right)$ with $k_{c}=$ $0.14 k$ enhances all three components on small scales and reduces the components on large scales. The relative velocity peak leaves a bump at $k \simeq 0.02 h \mathrm{Mpc}^{-1}$ in the squeezed configuration, similar to the scale seen in the equilateral case. Therefore, the unique signature of the relative velocity effect in the galaxy bispectrum on large scales can be used to robustly measure the relative velocity effect for $b_{r} \geq 0.01$ in low-redshift massive galaxies.

\section{Effect on BAO measurements: Power spectrum}

In addition to generating a large-scale bispectrum, the relative velocity effect can also modify the galaxy power spectrum on scales of order the sound horizon. Numerous ongoing and future galaxy surveys seek to measure the galaxy power spectrum on these scales with extraordinary precision to localize the BAO feature and thereby reconstruct the expansion history of the universe, constraining the kinematic properties of dark energy. From the viewpoint of BAO probes of dark energy, the relative velocity effect could be a significant contaminant, as we illustrate in this section.

\subsection{Relative velocity contribution and galaxy power spectrum}

We compute the power spectrum of the galaxy fluctuation using eq. (3.9), but for simplicity we first compute the relative velocity contributions only. With caution that the cross-power spectrum $P_{\times}^{i}(k)$ in eq. (3.5) depends 
on the direction of the wavevector, the ensemble average of the galaxy fluctuation is

$$
\begin{aligned}
\left.\left\langle\delta_{g}\left(\mathbf{k}_{a}\right) \delta_{g}\left(\mathbf{k}_{b}\right)\right)\right\rangle= & b_{r} \int \frac{d^{3} \mathbf{q}_{1}}{(2 \pi)^{3}} \int \frac{d^{3} \mathbf{q}_{2}}{(2 \pi)^{3}} \sum_{i=x, y, z}\left[2 b_{1} F_{2}\left(\mathbf{q}_{1}, \mathbf{k}_{a}-\mathbf{q}_{1}\right)\left\langle\delta_{m}\left(\mathbf{q}_{1}\right) \delta_{m}\left(\mathbf{k}_{a}-\mathbf{q}_{1}\right) u_{r}^{i}\left(\mathbf{q}_{2}\right) u_{r}^{i}\left(\mathbf{k}_{b}-\mathbf{q}\right)\right\rangle\right. \\
& \left.+b_{2}\left\langle\delta_{m}\left(\mathbf{q}_{1}\right) \delta_{m}\left(\mathbf{k}_{a}-\mathbf{q}_{1}\right) u_{r}^{i}\left(\mathbf{q}_{2}\right) u_{r}^{i}\left(\mathbf{k}_{b}-\mathbf{q}\right)\right\rangle\right] \\
& +b_{r}^{2} \int \frac{d^{3} \mathbf{q}_{1}}{(2 \pi)^{3}} \int \frac{d^{3} \mathbf{q}_{2}}{(2 \pi)^{3}} \sum_{i, j=x, y, z}\left\langle u_{r}^{i}\left(\mathbf{q}_{1}\right) u_{r}^{i}\left(\mathbf{k}_{a}-\mathbf{q}_{1}\right) u_{r}^{j}\left(\mathbf{q}_{2}\right) u_{r}^{j}\left(\mathbf{k}_{b}-\mathbf{q}\right)\right\rangle
\end{aligned}
$$

and by isolating the connected part only the galaxy power spectrum can be computed as

$$
\begin{aligned}
P_{g}(\mathbf{k}) & =b_{r} \int \frac{d^{3} \mathbf{q}}{(2 \pi)^{3}} \sum_{i=x, y, z} P_{\times}^{i}(\mathbf{q}) P_{\times}^{i}(\mathbf{k}-\mathbf{q})\left[4 b_{1} F_{2}(\mathbf{q}, \mathbf{k}-\mathbf{q})+2 b_{2}\right]+2 b_{r}^{2} \int \frac{d^{3} \mathbf{q}}{(2 \pi)^{3}} \sum_{i, j=x, y, z} P_{u}^{i j}(\mathbf{q}) P_{u}^{i j}(\mathbf{k}-\mathbf{q}) \\
& =\int \frac{d^{3} \mathbf{q}}{(2 \pi)^{3}} P_{m}(q) P_{m}(|\mathbf{k}-\mathbf{q}|) G_{u}(\mathbf{q}, \mathbf{k}-\mathbf{q})\left[4 b_{1} b_{r} F_{2}(\mathbf{q}, \mathbf{k}-\mathbf{q})+2 b_{2} b_{r}+2 b_{r}^{2} G_{u}(\mathbf{q}, \mathbf{k}-\mathbf{q})\right]
\end{aligned}
$$

(see Appendix A for derivation). The third term in the square-bracket is the pure relative velocity contribution to the galaxy power spectrum, while the other terms represent the contributions of the relative velocity effect in conjunction with the nonlinear galaxy bias.

Finally, accounting for all the remaining contributions from the nonlinear evolution in the matter density distribution, the full power spectrum of the galaxy fluctuation can be written as

$$
\begin{aligned}
P_{g}(\mathbf{k})= & b_{1}^{2} P_{\mathrm{NL}}(\mathbf{k})+\int \frac{d^{3} \mathbf{q}}{(2 \pi)^{3}} P_{m}(q) P_{m}(|\mathbf{k}-\mathbf{q}|)\left[\frac{1}{2} b_{2}^{2}+2 b_{1} b_{2} F_{2}(\mathbf{q}, \mathbf{k}-\mathbf{q})\right. \\
& \left.+4 b_{1} b_{r} F_{2}(\mathbf{q}, \mathbf{k}-\mathbf{q}) G_{u}(\mathbf{q}, \mathbf{k}-\mathbf{q})+2 b_{2} b_{r} G_{u}(\mathbf{q}, \mathbf{k}-\mathbf{q})+2 b_{r}^{2} G_{u}(\mathbf{q}, \mathbf{k}-\mathbf{q})^{2}\right] .
\end{aligned}
$$

To second order in the power spectrum, the linear bias parameter $b_{1}$ is renormalized. $P_{\mathrm{NL}}(k)$ in the first term is the nonlinear matter power spectrum, while $P_{m}(k)$ in the integral is computed by using the linear matter power spectrum [28, 29]. Compared to eq. (4.1), two additional contributions in the square-bracket arise from the nonlinear galaxy bias.

Figure 4 shows the full galaxy power spectrum computed by using eq. (4.2). Assuming our fiducial bias parameters $b_{2} / b_{1}=0.1$ and $b_{r} / b_{1}=0.01$, the galaxy power spectrum (thick solid) is largely determined by the matter power spectrum (linear: dotted, nonlinear: short dashed) on all scales, yet the relative velocity effect and the nonlinear galaxy bias affect the galaxy power spectrum at the percent level on various scales. These contributions expressed in the second square-bracket of eq. (4.2) are split into three components with different dependences on the relative velocity bias $b_{r}$ : The auto-power spectrum of the relative velocity effect $\left(b_{r}^{2}\right.$ : thin solid), the cross-power spectrum of the relative velocity and the nonlinear galaxy bias $\left(b_{r}\right.$ : long dashed), and the auto-power spectrum of the nonlinear galaxy bias (dot-dashed). The auto-power spectrum (thin solid) of the relative velocity effect closely resembles the velocity power spectrum with the prominent oscillation structure seen in Fig. 1a. The auto-power spectrum (dot-dashed) of the nonlinear galaxy bias is constant on large scales and approaches the shape of the matter power spectrum on small scales, while its power is enhanced at $k \simeq 0.2 h \mathrm{Mpc}^{-1}$ by the nonlinear kernel $F_{2}(\mathbf{q}, \mathbf{k}-\mathbf{q})$ that puts more weight on the large-scale power in the convolution. The cross-power spectrum (long dashed) of the relative velocity and the nonlinear galaxy bias changes sign at $k \simeq 0.04 \mathrm{~h} \mathrm{Mpc}^{-1}$, reflecting the out-of-phase nature of the velocity and the matter distributions seen in Fig. $1 b$.

Future galaxy surveys that use BAO probes of dark energy will measure the large-scale galaxy power spectrum and attempt to determine the BAO peak position at sub-percent level precision. At $k \simeq 0.1 \mathrm{hMpc}^{-1}$, the auto-power spectrum of the relative velocity effect (thin solid) is rather sub-dominant compared to the contribution of the cross term and the nonlinear galaxy bias term (long dashed and dot-dashed) for our fiducial parameters. Therefore, both the nonlinear bias $b_{2}$ and the relative velocity bias $b_{r}$ parameters must be determined well in order to completely clean out the relative velocity effect in the galaxy power spectrum. This trend remains unchanged for $b_{r} / b_{1}<0.1$ given $b_{2} / b_{1}=0.1$. 


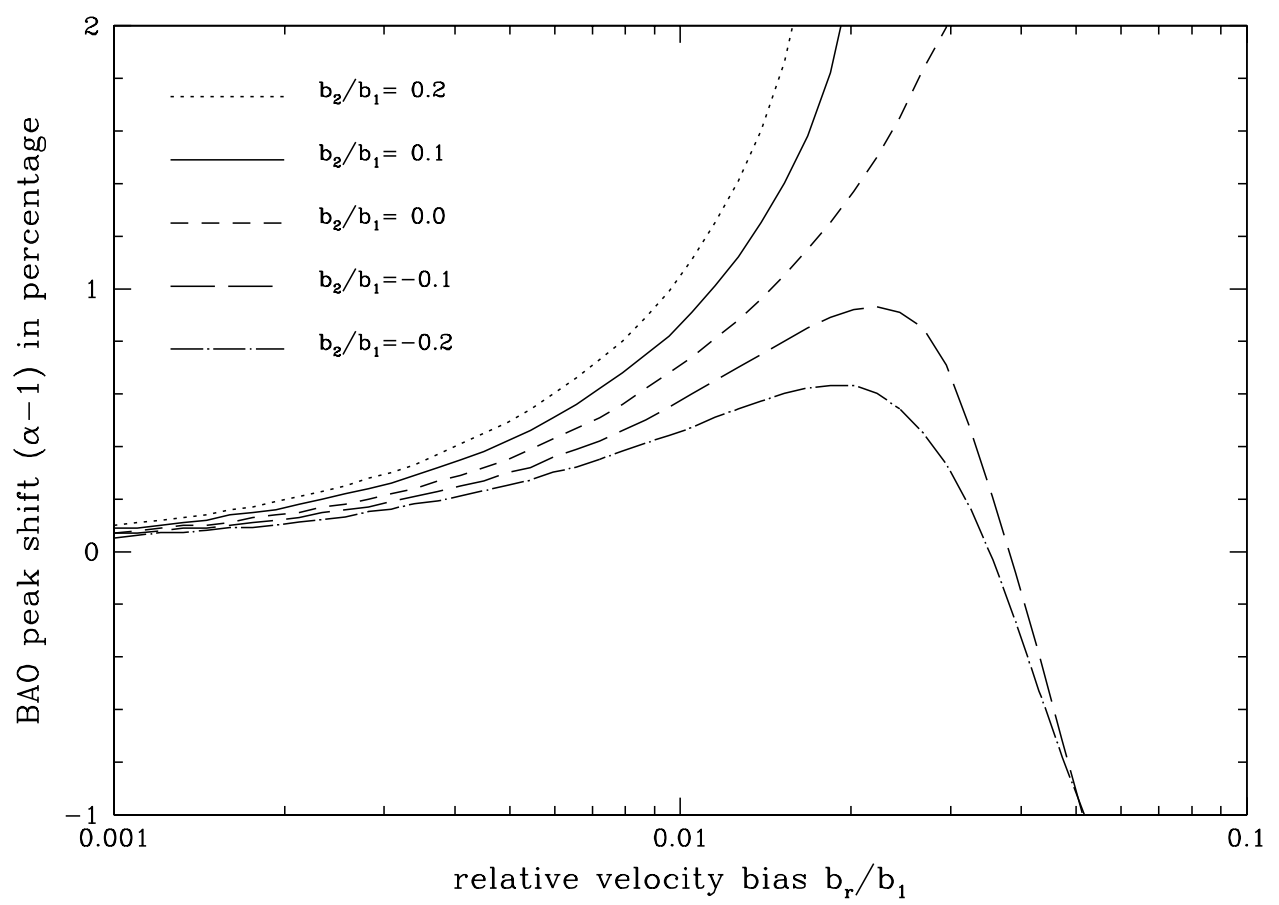

Figure 5. Impact of the relative velocity effect on the BAO peak shift. Various lines represent the peak shift $(\alpha-1)$ in percentage as a function of the relative velocity bias parameter $b_{r} / b_{1}$, given the value of the nonlinear galaxy bias parameter $b_{2} / b_{1}$. Following the Seo et al. [12] approach, the peak shift is obtained by fitting the template power spectrum to the full galaxy power spectrum in eq. (4.2) accounting for the nonlinear growth and the anomalous power (see text for detail).

\subsection{Shift in the BAO peak position}

We quantify the impact of the relative velocity effect on the precision measurement of the baryonic acoustic oscillation feature in future dark energy surveys, assuming that no information is utilized for cleaning out the relative velocity effect in the power spectrum measurement. Following the Seo et al. [12] approach to modeling the scale-dependent nonlinear growth and anomalous power in the matter power spectrum, we fit the template power spectrum to the full galaxy power spectrum computed in eq. (4.2) to characterize the BAO peak shift. The template power spectrum is parametrized as

$$
P_{t}(k)=\left(c_{0}+c_{1} k+c_{2} k^{2}\right) P_{\mathrm{evo}}(k / \alpha)+\left(a_{0}+a_{1} k+a_{2} k^{2}+\cdots+a_{7} k^{7}\right)
$$

with polynomial coefficients $c_{i}$ and $a_{i}$ taken as free parameters. Any deviation of the parameter $\alpha$ from unity represents the BAO peak shift in measurements. The two groups of polynomials in $k$ (multiplicative and additive) account for the scale-dependent nonlinear growth and the additive broad-band power; This parametrization corresponds to the "Poly7" fit in Seo et al. [12]. The evolved linear matter power spectrum is then given in the form

$$
P_{\text {evo }}(k)=\left[P_{\text {lin }}(k)-P_{\text {no-wiggle }}(k)\right] \exp \left(-k^{2} \Sigma_{m}^{2} / 2\right)+P_{\text {no-wiggle }}(k),
$$

where $P_{\text {no-wiggle }}(k)$ is the fit to the smooth power spectrum shape without the BAO wiggle from Eisenstein and $\mathrm{Hu}$ [24] and $\Sigma_{m}=8.8 h^{-1} \mathrm{Mpc}$ accounts for the degradation of the BAO wiggle in time $(z=0)$ [12].

In Fig. 5 we perform a $\chi^{2}$ analysis to compute the BAO peak shift as a function of the bias parameters $b_{r} / b_{1}$ and $b_{2} / b_{1}$ by fitting the template power spectrum in eq. (4.3) to the full galaxy power spectrum in eq. (4.2) over a range of $0.02 h \mathrm{Mpc}^{-1}<k<0.35 h \mathrm{Mpc}^{-1}$. Since nonlinear effects do shift the BAO peak position $(\sim 0.5 \%$ at $z=0.3$ [12]) at low redshift, we isolate the effect of the relative velocity on the BAO peak shift by setting $\Delta \alpha=0$ when $b_{r} / b_{1}=0$. The relative velocity effect shifts the BAO peak position no more than $1 \%$ at $b_{r} / b_{1} \leq 0.01$ with a reasonable range of the nonlinear galaxy bias parameter, but its impact increases dramatically at $b_{r} / b_{1} \gg 0.01$, because the acoustic structure in the relative velocity effect is anticorrelated with the structure in the matter distribution. While a large nonlinear galaxy bias can affect the BAO 
peak position, its parameters are, by contrast, relatively well constrained, and its impact is at the sub-percent level for our fiducial value $b_{r} / b_{1}$ over a range of $b_{2} / b_{1}$ values considered here. For negative values of $b_{2} / b_{1}$, the cross-power spectrum (long dashed in Fig. 4) of the nonlinear galaxy bias becomes positive, but the autopower spectrum (dot-dashed in Fig. 4) of the nonlinear galaxy bias is reduced due to the sign change in $b_{2}$. At $k \geq 0.1 \mathrm{~h} \mathrm{Mpc}^{-1}$, the latter is dominant over the cross-power spectrum, and hence the peak shift is reduced at $b_{r} / b_{1}=0.01$, compared to the case with $b_{2} / b_{1}>0$. However, as the relative velocity bias increases, the cross-power spectrum contributes more to the peak shift, changing its direction. The auto-power spectrum (thin solid in Fig. 4) of the pure relative velocity contribution around the BAO scale is rather sub-dominant over the scales for the range of $b_{r} / b_{1}$ considered here.

\section{Cosmological constraining power and bias in parameter estimation}

In section 4 we showed that the presence of the relative velocity effect can adversely affect our ability to measure the BAO peak position and constrain the cosmological parameters when its effect is ignored in the power spectrum analysis. However, it was also demonstrated in section 3 that measurement of the bispectrum provides a promising way to measure the relative velocity effect and account for it when we estimate the cosmological parameters. In this section, we perform a Fisher matrix analysis to answer three questions: What is the bias in our parameter estimation if the relative velocity effect is unaccounted for? How well can we measure or constrain the relative velocity effect using the galaxy power spectrum and bispectrum measurements? And how much would the cosmological parameter constraints be inflated if we included the relative velocity bias as additional parameter? To answer these questions and for definiteness, we consider a galaxy survey of volume $V=10\left(h^{-1} \mathrm{Gpc}\right)^{3}$ with number density $n_{g}=10^{-4}\left(h^{-1} \mathrm{Mpc}\right)^{3}$. As our cosmological model, we assume a flat universe with a constant dark energy equation-of-state and add two bias parameters $b_{2} / b_{1}$ and $b_{r} / b_{1}$ to describe the nonlinear galaxy bias and the relative velocity distribution: $\mathbf{p}=\left(n_{s}, \alpha_{s}, \omega_{m}, \omega_{b}, \omega_{\mathrm{de}}, w_{0}, b_{2} / b_{1}, b_{r} / b_{1}\right)$ with $h^{2}=\omega_{m}+\omega_{\mathrm{de}}$ (see section 1 for our choice of the fiducial model parameters). ${ }^{1}$

First, we compute the impact of the relative velocity effect on cosmological parameter estimation from galaxy power spectrum measurements when the relative velocity effect is unaccounted. This case will represent the currently planned galaxy surveys, as the null hypothesis is devoid of the relative velocity effect. Here we quantify its impact in terms of the parameter bias $\Delta w_{0}$ in the dark energy equation-of-state. Our model parameters are described by $\overline{\mathbf{p}} \equiv \mathbf{p}\left[b_{r} / b_{1}=0\right]$, in which the relative velocity bias parameter is incorrectly set zero $\left(b_{r}=0\right)$, while in reality it is nonzero $\left(b_{r} \neq 0\right)$. The parameter bias in our estimation of the dark energy equation-of-state is then (see Appendix B)

$$
\Delta w_{0}=\sum_{i}^{\overline{\mathbf{p}}}\left[F^{-1}(\overline{\mathbf{p}})\right]_{w_{0} i} \times \sum_{k=k_{\min }}^{k_{\max }} \frac{1}{\sigma_{P_{g}}^{2}(k)} \frac{\partial P_{g}(k)}{\partial p_{i}}\left[P_{g}(k \mid \mathbf{p})-P_{g}(k \mid \overline{\mathbf{p}})\right]
$$

where the Fisher information matrix is

$$
F_{i j}(\overline{\mathbf{p}})=\sum_{k=k_{\min }}^{k_{\max }} \frac{1}{\sigma_{P_{g}}^{2}(k)} \frac{\partial P_{g}(k \mid \overline{\mathbf{p}})}{\partial p_{i}} \frac{\partial P_{g}(k \mid \overline{\mathbf{p}})}{\partial p_{j}}
$$

the uncertainty in the power spectrum measurements is [30]

$$
\sigma_{P_{g}}^{2}(k)=\frac{2}{4 \pi^{2} k^{2} \Delta k V /(2 \pi)^{3}}\left[P_{g}(k)+\frac{1}{n_{g}}\right]^{2},
$$

and $k_{\min }=\Delta k=2 \pi / V^{1 / 3}=0.0029 \mathrm{hMpc}^{-1}$. In computing the Fisher information matrix in eq. (5.2) we also add the Planck prior on the cosmological parameters, following the procedure described in the Appendix of the Dark Energy Task Force final report [1] (see also [31, 32]).

\footnotetext{
${ }^{1}$ We assume that the linear bias $b_{1}$ and the matter fluctuation normalization $\Delta_{\varphi}^{2}$ is degenerate in the power spectrum analysis, and we can only constrain its combination. The degeneracy is partially broken by the nonlinear effect and also by the bispectrum, but since the normalization is nuisance in the present analysis we combine it with the linear bias term and remove it from our parameter set by assuming that other parameters are not affected by the change in the overall normalization of the measurements.
} 

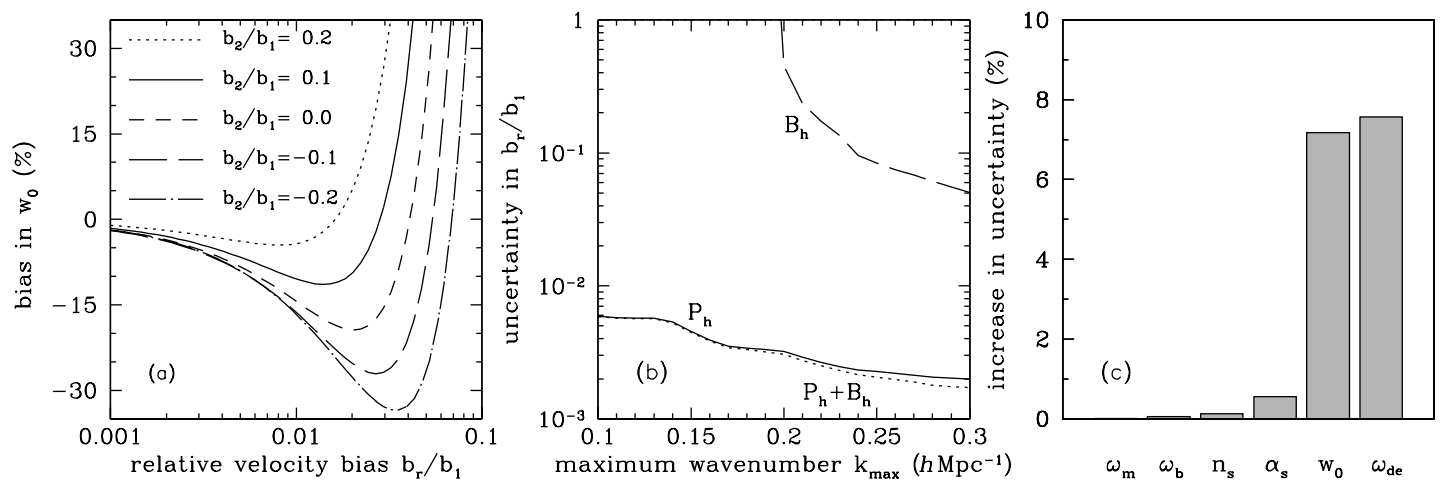

Figure 6. Impact of the relative velocity effect on the cosmological parameter estimation. For computing the constraint on the dark energy equation-of-state $w_{0}$, we use the galaxy power spectrum measurements in a survey of volume $V=$ $10\left(h^{-1} \mathrm{Gpc}\right)^{3}$ with number density $n_{g}=10^{-4}\left(h^{-1} \mathrm{Mpc}\right)^{-3}$. Parameter constraints are obtained by adding CMB priors from Planck and marginalizing over the remaining parameters (see text for details). (a) Parameter bias in measuring $w_{0}$ when the relative velocity effect is unaccounted. (b) Measurement uncertainty in the relative velocity bias parameter (the nonlinear galaxy bias parameter is fixed $b_{2} / b_{1}=0.1$ ). (c) Fractional increase in the cosmological parameter constraints when the relative velocity bias is modeled and marginalized over $\left(k_{\max }=0.2 \mathrm{hMpc}^{-1}\right)$.

Figure 6a shows the bias in the dark energy equation-of-state $w_{0}$ as a function of the relative velocity bias given various values of the nonlinear galaxy bias $b_{2} / b_{1}$. We have fixed $k_{\max }=0.2 h \mathrm{Mpc}^{-1}$. Naturally, the bias $\Delta w_{0}$ increases with larger value of $b_{r} / b_{1}$, as the relative velocity effect is unaccounted for. Negative values of $b_{2} / b_{1}$ reduce the auto-power spectrum (dot-dashed in Fig. 4 ) of the nonlinear galaxy bias and change the sign of its cross-power spectrum (long dashed in Fig. 4) with the relative velocity on small scales, enhancing the difference in the power spectrum around the BAO scale and thereby increasing the bias $\Delta w_{0}$, compared to reducing the relative velocity contribution in the fiducial model with positive $b_{2} / b_{1}$. Meanwhile, since our parameter estimation is affected by the power spectrum measurements on all scales rather than just on BAO scales, there is a cross-over point in $b_{r} / b_{1}$, at which the bias from the cross-power spectrum is cancelled by the bias from the auto-power spectrum of the relative velocity. Moreover, the parameter bias obtained here is rather insensitive to the precision of the power spectrum measurements, since it arises from our incorrect modeling of the mean value of the power spectrum. For the fiducial value $b_{2} / b_{1}=0.1$ (solid), the bias $\Delta w_{0}$ is at the $10 \%$ level for $b_{r} / b_{1}=0.01$ and $2 \%$ for $b_{r} / b_{1}=0.001$. While a more thorough analysis of the BAO peak position may further reduce the parameter bias than our simple estimates using the Fisher matrix (like in section 4.2, e.g., [12, 33]), Fig. 6a demonstrates the significance of the relative velocity effect on the future dark energy surveys, and the relative velocity bias parameter should be included and marginalized over in the power spectrum analysis.

Next, we estimate our ability to measure the relative velocity effect from the galaxy power spectrum and bispectrum measurements. Our fiducial model in this case is then described by $\mathbf{p}\left[b_{r} / b_{1}=0\right]$, correctly representing the reality. The measurement uncertainty in the relative velocity bias parameter can be expressed as

$$
\sigma_{b_{r} / b_{1}}^{2}=\left[F^{-1}(\mathbf{p})\right]_{b_{r} / b_{1} b_{r} / b_{1}},
$$

and the Fisher matrix $F_{i j}$ now includes that of the bispectrum when the bispectrum measurements are used 
[34-36],

$$
F_{i j}=\sum_{k_{a}=k_{\min }}^{k_{\max }} \sum_{k_{b}=k_{\min }}^{k_{a}} \sum_{k_{c}=k_{\min }^{\star}}^{k_{b}} \frac{1}{\sigma_{B_{g}}^{2}} \frac{\partial B_{g}\left(k_{a}, k_{b}, k_{c}\right)}{\partial p_{i}} \frac{\partial B_{g}\left(k_{a}, k_{b}, k_{c}\right)}{\partial p_{j}}
$$

with the bispectrum variance

$$
\sigma_{B_{g}}^{2}\left(k_{a}, k_{b}, k_{c}\right)=\frac{s_{a b c}}{8 \pi^{2} k_{a} k_{b} k_{c} \Delta k^{3} V /(2 \pi)^{3}}\left[P_{g}\left(k_{a}\right)+\frac{1}{n_{g}}\right]\left[P_{g}\left(k_{b}\right)+\frac{1}{n_{g}}\right]\left[P_{g}\left(k_{c}\right)+\frac{1}{n_{g}}\right],
$$

where $k_{\min }^{\star}=\max \left(k_{\min },\left|k_{a}-k_{b}\right|\right)$ and the symmetry factor is $s_{a b c}=6,2,1$ for equilateral, isosceles, and general triangular configurations, respectively. We have assumed that the bispectrum estimates are Gaussian distributed.

Figure $6 \mathrm{~b}$ shows the measurement uncertainty $\sigma_{b_{r} / b_{1}}$ in the relative velocity bias parameter, after all the remaining parameters $\mathbf{p}$ are marginalized over, which can be translated into the detection significance $S / N \simeq\left(b_{r} / b_{1}\right) / \sigma_{\left(b_{r} / b_{1}\right)}$. With the few percent level contribution of the relative velocity effect at $b_{r} / b_{1}=0.01$ around the BAO scale seen in Fig. 4, power spectrum measurements (solid) alone can constrain the relative velocity bias as small as $b_{r} / b_{1}=0.01$ at the $2-3 \sigma$ confidence level. Bispectrum measurements (dashed) provide additional but less stringent constraint on the relative velocity bias parameter if $b_{r}=0$, than power spectrum measurements, especially when the basic cosmological parameters are already well constrained by CMB measurements. Hence, the combination (dotted) of the power spectrum and bispectrum measurements yields constraints mainly derived from the power spectrum measurements. However, as illustrated in Fig. 3, the bispectrum exhibits a unique signature of the relative velocity effect on large scales, if the relative velocity effect is present. Therefore, if any hint of the relative velocity effect is found in the power spectrum analysis, measurements of the bispectrum can be used to confirm and constrain the relative velocity effect in an indisputable way.

Finally, we investigate the cost in the parameter constraints by adding the additional parameter $b_{r} / b_{1}$ and marginalizing over it. Figure $6 \mathrm{c}$ compares the parameter constraints in two cases, when the relative velocity bias parameter is modeled, and when it is simply assumed zero, i.e., $\sigma_{p_{\alpha}}(\mathbf{p}) / \sigma_{p_{\alpha}}(\overline{\mathbf{p}})$. The matter and the baryon density constraints $\sigma_{\omega_{m}}$ and $\sigma_{\omega_{b}}$ are little affected by the addition of the relative velocity bias parameter, as they are largely independent. The constraints on the spectral index $n_{s}$ and its running $\alpha_{s}$ become weaker by a few percent level, compared to the constraints in the model without the relative velocity bias parameter, indicating that the relative velocity effect is somewhat degenerate with $n_{s}$ and $\alpha_{s}$. Last, as we showed in previous sections the relative velocity effect can adversely affect the dark energy parameter estimation, and Fig. 6c shows that the constraints on dark energy parameters $w_{0}$ and $\omega_{\text {de }}$ would degrade by $\sim 8 \%$, if we included the relative velocity bias parameter. While the spectral index and its running are also affected by the relative velocity parameter, they are highly constrained by CMB measurements, in contrast to the dark energy parameters. Meanwhile, the inflated constraints on the dark energy parameters can be reduced by using the bispectrum measurement to improve the constraint on the relative velocity bias parameter and thereby recovering the dark energy constraints. Overall, the cost of modeling the relative velocity bias is rather low, especially considering the magnitude of the parameter bias when the relative velocity effect is unaccounted for.

\section{Discussion}

We have investigated the relative velocity effect of the baryon and the dark matter distributions on the baryonic acoustic oscillation (BAO) measurements at low redshift. The relative velocity effect imprinted in the earliest baryonic structure at high redshift leaves its signature in the power spectrum of these objects [16], which in turn alters the subsequent star formation and reionization history, affecting the large-scale clustering of massive galaxies at low redshift. The relative velocity effect that persists until the late time results in a nonvanishing galaxy bispectrum, and its contribution to the bispectrum can be easily isolated on large scales $k \simeq 0.03 \mathrm{~h} \mathrm{Mpc}^{-1}$, where the relative velocity contribution peaks. Since the acoustic structure of the relative velocity effect is non-smooth and out-of-phase compared to the structure in the matter distribution, its effect cannot be removed by using a broad-band filter that is designed to deal with the nonlinear matter evolution, and the relative velocity effect can potentially bias the BAO peak position determination. 
We find that the relative velocity effect can, if unaccounted, shift the BAO peak position measurements and bias the estimates of the dark energy equation-of-state by $\sim 10 \%$, depending on the amplitude of the relative velocity effect. However, the power spectrum measurement itself is sensitive enough to the relative velocity effect that we can measure its amplitudes as small as $b_{r} / b_{1} \simeq 0.01$ along with other cosmological parameters. Including the relative velocity bias as additional parameter may inflate the constraint $\sigma_{w_{0}}$ on the dark energy equation-of-state by $8 \%$, a marginal cost to pay, compared to the potentially pernicious risk arising from the unaccounted relative velocity effect. Bispectrum measurements also provide tight constraints on the relative velocity effect, though its constraint in the fiducial model without the relative velocity effect is less stringent than from power spectrum measurements due to larger measurement uncertainties. However, the bispectrum signal is substantially enhanced, especially on large scales, if the relative velocity effect is present, providing alternative way to confirm and measure the relative velocity effect in a more robust and model-independent way.

Many future dark energy surveys such as BigBoss, ${ }^{2}$ Euclid, ${ }^{3}$ and the Wide-Field Infrared Survey Telescope $^{4}$ plan on measuring galaxies at moderately high redshift, $z \gtrsim 1$, where nonlinear growth is less severe and even smaller scales may safely be included in the analysis. However, these high redshift surveys are not immune to the relative velocity effect, which grows in amplitude like $(1+z)$, eventually dominating over the matter fluctuations at $z \gg 10$. At the redshifts probed by these BAO surveys, the largest uncertainty in our calculation is the amplitude of the relative velocity bias parameter $b_{r}$. Any effect of relative velocities on massive galaxies at such redshifts must be indirect, making difficult any theoretical estimation of the relative velocity bias parameter. It is quite plausible that the complicated process of massive galaxy formation obliterates the relative velocity effect at low redshift, meaning that this effect may not be apparent in the distribution of low-redshift massive galaxies. Nevertheless, we note that the relative velocity effect can be easily modeled without significantly inflating the cosmological parameter constraints, and if present but unaccounted, it can catastrophically affect the ability of BAO survey to perform high precision cosmological parameter estimation.

\section{Acknowledgments}

We thank Tobias Baldauf, Chris Hirata, Hee-Jong Seo, and Dmitriy Tseliakhovich for useful discussions. J.Y. is supported by the SNF Ambizione Grant. This work is supported by the Swiss National Foundation under contract 200021-116696/1 and WCU grant R32-10130.

\section{References}

[1] A. Albrecht et al., ArXiv Astrophysics e-prints (2006), astro-ph/ 0609591.

[2] E. Komatsu, K. M. Smith, J. Dunkley, C. L. Bennett, B. Gold, G. Hinshaw, N. Jarosik, D. Larson, M. R. Nolta, L. Page, et al., Astrophys. J. Suppl. Ser. 192, 18 (2011), 1001.4538.

[3] D. Larson, J. Dunkley, G. Hinshaw, E. Komatsu, M. R. Nolta, C. L. Bennett, B. Gold, M. Halpern, R. S. Hill, N. Jarosik, et al., Astrophys. J. Suppl. Ser. 192, 16 (2011), 1001.4635.

[4] H.-J. Seo and D. J. Eisenstein, Astrophys. J. 633, 575 (2005), arXiv: astro-ph/ 0507338.

[5] D. Jeong and E. Komatsu, Astrophys. J. 651, 619 (2006), arXiv: astro-ph / 0604075.

[6] D. J. Eisenstein, H.-J. Seo, and M. White, Astrophys. J. 664, 660 (2007), arXiv : astro-ph/ 0604361.

[7] M. Crocce and R. Scoccimarro, Phys. Rev. D 77, 023533 (2008), arXiv: 0704.2783.

[8] R. E. Smith, R. Scoccimarro, and R. K. Sheth, Phys. Rev. D 77, 043525 (2008), arXiv : astro-ph / 0703620.

[9] A. Taruya, T. Nishimichi, and S. Saito, Phys. Rev. D 82, 063522 (2010), 1006.0699.

[10] V. Desjacques, M. Crocce, R. Scoccimarro, and R. K. Sheth, Phys. Rev. D 82, 103529 (2010), 1009.3449.

[11] C. Orban and D. H. Weinberg, ArXiv e-prints (2011), 1101.1523.

\footnotetext{
${ }^{2}$ http://bigboss.lbl.gov

${ }^{3}$ http://sci.esa.int/euclid

${ }^{4}$ http://wfirst.gsfc.nasa.gov
} 
[12] H.-J. Seo, E. R. Siegel, D. J. Eisenstein, and M. White, Astrophys. J. 686, 13 (2008), 0805.0117.

[13] A. G. Sánchez, C. M. Baugh, and R. Angulo, Mon. Not. R. Astron. Soc. 390, 1470 (2008), 0804 . 0233.

[14] J. Yoo and J. Miralda-Escudé, Phys. Rev. D 82, 043527 (2010), 0901.0708.

[15] D. Tseliakhovich and C. Hirata, Phys. Rev. D 82, 083520 (2010), 1005.2416.

[16] N. Dalal, U. Pen, and U. Seljak, J. Cosmol. Astropart. Phys. 11, 7 (2010), 1009.4704.

[17] D. Tseliakhovich, R. Barkana, and C. Hirata, ArXiv e-prints (2010), 1012.2574.

[18] T. Greif, S. White, R. Klessen, and V. Springel, ArXiv e-prints (2011), 1101.5493.

[19] U. Maio, L. V. E. Koopmans, and B. Ciardi, Mon. Not. R. Astron. Soc. pp. L197+ (2011), 1011.4006.

[20] A. Stacy, V. Bromm, and A. Loeb, ArXiv e-prints (2010), 1011.4512.

[21] M. Tegmark and et al., Phys. Rev. D 74, 123507 (2006), arXiv: astro-ph/ 0608632.

[22] B. A. Reid, W. J. Percival, D. J. Eisenstein, L. Verde, D. N. Spergel, R. A. Skibba, N. A. Bahcall, T. Budavari, M. Fukugita, J. R. Gott, et al., ArXiv e-prints (2009), 0907.1659.

[23] U. Seljak and M. Zaldarriaga, Astrophys. J. 469, 437 (1996), astro-ph/9603033.

[24] D. J. Eisenstein and W. Hu, Astrophys. J. 496, 605 (1998), arXiv: astro-ph/ 9709112.

[25] M. H. Goroff, B. Grinstein, S. Rey, and M. B. Wise, Astrophys. J. 311, 6 (1986).

[26] B. Jain and E. Bertschinger, Astrophys. J. 431, 495 (1994), arXiv: astro-ph/ 9311070.

[27] N. Makino, M. Sasaki, and Y. Suto, Phys. Rev. D 46, 585 (1992).

[28] A. F. Heavens, S. Matarrese, and L. Verde, Mon. Not. R. Astron. Soc. 301, 797 (1998), arXiv:astro-ph/9808016.

[29] P. McDonald, Phys. Rev. D 74, 103512 (2006), arXiv: astro-ph/ 0609413.

[30] H. A. Feldman, N. Kaiser, and J. A. Peacock, Astrophys. J. 426, 23 (1994), arXiv: astro-ph/ 9304022.

[31] M. Zaldarriaga, D. N. Spergel, and U. Seljak, Astrophys. J. 488, 1 (1997), arXiv: astro-ph/ 9702157.

[32] J. R. Bond, G. Efstathiou, and M. Tegmark, Mon. Not. R. Astron. Soc. 291, L33 (1997), arXiv:astro-ph/9702100.

[33] N. Padmanabhan and M. White, Phys. Rev. D 77, 123540 (2008), 0804.0799.

[34] R. Scoccimarro, E. Sefusatti, and M. Zaldarriaga, Phys. Rev. D 69, 103513 (2004), arXiv:astro-ph/0312286.

[35] E. Sefusatti, M. Crocce, S. Pueblas, and R. Scoccimarro, Phys. Rev. D 74, 023522 (2006), arXiv:astro-ph/0604505.

[36] T. Baldauf, U. Seljak, and L. Senatore, ArXiv e-prints (2010), 1011.1513.

[37] M. Tegmark, A. N. Taylor, and A. F. Heavens, Astrophys. J. 480, 22 (1997), arXiv : astro-ph/ 9603021.

[38] H. Wu, E. Rozo, and R. H. Wechsler, Astrophys. J. 688, 729 (2008), 0803.1491.

\section{A Power spectrum and bispectrum computation}

Here we derive key equations for computing the full galaxy power spectrum in eq. (4.2) and bispectrum in eq. (3.10). Full computation of the galaxy power spectrum requires a volume integration over the wavevector $\mathbf{q}$, 
and it can be obtained by a combination of the two integrations:

$$
\begin{aligned}
& \int \frac{d^{3} \mathbf{q}}{(2 \pi)^{3}} P_{m}(q) P_{m}(|\mathbf{k}-\mathbf{q}|) F_{2}(\mathbf{q}, \mathbf{k}-\mathbf{q}) \\
& \quad=\frac{k^{3}}{2 \pi^{2}} \int_{0}^{\infty} d r r^{2} P_{m}(k r) \int_{-1}^{1} d \mu P_{m}\left(k \sqrt{1+r^{2}-2 r \mu}\right)\left[\frac{3 r+7 \mu-10 r \mu^{2}}{14 r\left(1+r^{2}-2 r \mu\right)}\right] \\
& \int \frac{d^{3} \mathbf{q}}{(2 \pi)^{3}} P_{m}(q) P_{m}(|\mathbf{k}-\mathbf{q}|) G_{u}(\mathbf{q}, \mathbf{k}-\mathbf{q}) \\
& =\frac{k^{3}}{2 \pi^{2}} \int_{0}^{\infty} d r r^{2} P_{m}(k r) \int_{-1}^{1} d \mu P_{m}\left(k \sqrt{1+r^{2}-2 r \mu}\right)\left[\frac{T_{r u}(q)}{T_{m}(q)} \frac{T_{r u}\left(k \sqrt{1+r^{2}-2 r \mu}\right)}{T_{m}\left(k \sqrt{1+r^{2}-2 r \mu}\right)} \frac{r-\mu}{\sqrt{1+r^{2}-2 r \mu}}\right]
\end{aligned}
$$

where $r=q / k$ is the ratio of the two wavevectors $\mathbf{k}$ and $\mathbf{q}$ and $\mu$ is their cosine angle.

For the bispectrum computation, since the connected wavevectors $\left(\mathbf{k}_{a}+\mathbf{k}_{b}+\mathbf{k}_{c}=0\right)$ defines a $2 \mathrm{D}$ surface rather than a line $\left(\mathbf{k}_{a}+\mathbf{k}_{b}=0\right)$ in the power spectrum case, the azimuthal symmetry in the volume integration is broken, and a full 3D integration needs to be performed. Given a triangular configuration $\left(k_{a}, k_{b}\right.$, and $\mu_{a b}=\mathbf{k}_{a} \cdot \mathbf{k}_{b} / k_{a} k_{b}$ ), the last term of the full bispectrum in eq. (3.10) is

$$
\begin{gathered}
\int \frac{d^{3} \mathbf{q}}{(2 \pi)^{3}} P_{m}\left(\left|\mathbf{k}_{a}+\mathbf{q}\right|\right) P_{m}\left(\left|\mathbf{k}_{b}-\mathbf{q}\right|\right) P_{m}(q) G_{u}\left(\mathbf{k}_{a}+\mathbf{q}, \mathbf{k}_{b}-\mathbf{q}\right) G_{u}\left(\mathbf{k}_{a}+\mathbf{q}, \mathbf{q}\right) G_{u}\left(\mathbf{q}, \mathbf{k}_{b}-\mathbf{q}\right) \\
=-\frac{k^{3}}{2 \pi^{2}} \int_{0}^{\infty} d r r^{2} P_{m}(k r) \frac{T_{r u}^{2}(k r)}{T_{m}^{2}(k r)} \int_{-1}^{1} \frac{d \mu}{2} P_{m}\left(k_{a} \sqrt{1+r^{2}+2 r \mu}\right) \frac{T_{r u}^{2}\left(k_{a} \sqrt{1+r^{2}+2 r \mu}\right)}{T_{m}^{2}\left(k_{a} \sqrt{1+r^{2}+2 r \mu}\right)\left[\frac{\mu+r}{\sqrt{1+r^{2}+2 r \mu}}\right]} \\
\times \int_{0}^{2 \pi} \frac{d \phi}{2 \pi} P_{m}\left(k_{a} \sqrt{r^{2}+r^{\prime 2}-2 r r^{\prime} \mu_{b q}}\right) \frac{T_{r u}^{2}\left(k_{a} \sqrt{r^{2}+r^{\prime 2}-2 r r^{\prime} \mu_{b q}}\right)}{T_{m}^{2}\left(k_{a} \sqrt{r^{2}+r^{\prime 2}-2 r r^{\prime} \mu_{b q}}\right)} \\
\times \frac{r^{\prime} \mu_{a b}-r \mu+r r^{\prime} \mu_{b q}-r^{2}}{\sqrt{1+r^{2}+2 r \mu} \sqrt{r^{2}+r^{\prime 2}-2 r r^{\prime} \mu_{b q}}} \frac{r^{\prime} \mu_{b q}-r}{\sqrt{r^{2}+r^{\prime 2}-2 r r^{\prime} \mu_{b q}}}
\end{gathered}
$$

with $r^{\prime}=k_{b} / k_{a}$ and $\mu_{b q}=\mu_{a b} \mu+\sqrt{1-\mu_{a b}^{2}} \sqrt{1-\mu^{2}} \cos \phi$, where $\phi$ is the azimuthal angle of $\mathbf{q}$.

\section{B Parameter forecast and bias in the parameter estimation}

Assuming the measurements of the galaxy power spectrum and bispectrum are Gaussian distributed, we can compute the likelihood of the measurements given a set of model parameters $\mathbf{p}$,

$$
2 \mathcal{L}(\mathbf{p})=\ln \operatorname{det} \mathbf{C}+(\mathbf{x}-\boldsymbol{\mu})^{T} \mathbf{C}^{-1}(\mathbf{x}-\boldsymbol{\mu})+\text { constant },
$$

where $\mathbf{x}$ and $\boldsymbol{\mu}(\mathbf{p})$ respectively represent the measurement and the model prediction of the galaxy power spectrum $P_{g}(k)$ and bispectrum $B_{g}\left(\mathbf{k}_{a}, \mathbf{k}_{b}, \mathbf{k}_{c}\right)$, and $\mathbf{C}(\mathbf{p})$ is the covariance matrix for the measurements. For forecasting the parameter constraints in galaxy surveys in section 5 we have used the Fisher information matrix (see, e.g., $[37,38])$

$$
F_{i j}=\left\langle\frac{\partial^{2} \mathcal{L}}{\partial p_{i} \partial p_{j}}\right\rangle=\frac{1}{2} \operatorname{Tr}\left[\mathbf{C}^{-1} \mathbf{C}_{, i} \mathbf{C}^{-1} \mathbf{C}_{, j}+2 \boldsymbol{\mu}_{, i}^{T} \mathbf{C}^{-1} \boldsymbol{\mu}_{, j}\right] \simeq \sum_{\mathbf{k}} \frac{1}{\sigma_{k}^{2}} \frac{\partial \mu(\mathbf{k})}{\partial p_{i}} \frac{\partial \mu(\mathbf{k})}{\partial p_{j}}
$$

where the first term involving the derivative of the covariance matrix is smaller than the other, and hence we ignored the derivatives of the covariance matrix in computing the constraints.

When the assumed fiducial model is incorrect, our computation of the mean and its covariance matrix will misrepresent the measurements, providing biased best-fit parameters $\mathbf{p}$ different from the true parameters $\mathbf{p}_{t}$. Assuming that our model is not far off from the true model, the best-fit parameters can be approximated as $\mathbf{p}=\mathbf{p}_{t}+\delta \mathbf{p}$, and the bias $\delta \mathbf{p}$ in the parameter estimation can be obtained from the relation that the best-fit 
parameters $\mathbf{p}$ maximize the likelihood in eq. (B.1),

$$
\begin{aligned}
0 & =\left\langle\frac{\partial \mathcal{L}}{\partial p_{i}}(\mathbf{p})\right\rangle=\operatorname{Tr}\left[\mathbf{C}^{-1} \mathbf{C}_{, i}-\mathbf{C}^{-1} \mathbf{C}_{, i} \mathbf{C}^{-1}\langle\mathbf{D}\rangle+\mathbf{C}^{-1}\left\langle\mathbf{D}_{, i}\right\rangle\right] \\
& =\operatorname{Tr}\left[\mathbf{C}^{-1} \mathbf{C}_{, i} \mathbf{C}^{-1}\left(\mathbf{C}-\mathbf{C}_{t}\right)-2 \boldsymbol{\delta} \boldsymbol{\mu}^{T} \mathbf{C}^{-1} \boldsymbol{\mu}_{, i}\right]+2 \sum_{j} \delta p_{j} \operatorname{Tr}\left[\frac{1}{2} \mathbf{C}^{-1} \mathbf{C}_{, i} \mathbf{C}^{-1} \mathbf{C}_{, j}+\boldsymbol{\mu}_{, j}^{T} \mathbf{C}^{-1} \boldsymbol{\mu}_{, i}\right] .
\end{aligned}
$$

The data matrix is $\mathbf{D}=(\mathbf{x}-\boldsymbol{\mu})(\mathbf{x}-\boldsymbol{\mu})^{T}$, and the ensemble averages of the data matrix and its derivative are

$$
\begin{aligned}
\langle\mathbf{D}\rangle & =\mathbf{C}_{t}\left(\mathbf{p}_{t}\right)+\left(\boldsymbol{\mu}_{t}-\boldsymbol{\mu}\right)\left(\boldsymbol{\mu}_{t}-\boldsymbol{\mu}\right)^{T} \simeq \mathbf{C}_{t}-\sum_{j} \mathbf{C}_{, j} \delta p_{j}+\mathcal{O}\left(\delta p^{2}\right), \\
\left\langle\mathbf{D}_{, i}\right\rangle & =2 \boldsymbol{\mu}_{, i} \sum_{j} \boldsymbol{\mu}_{, j}^{T} \delta p_{j}-2 \boldsymbol{\mu}_{, i} \boldsymbol{\delta} \boldsymbol{\mu}^{T},
\end{aligned}
$$

where $\mathbf{C}_{t}$ is the true covariance matrix and the mean is related to the true mean as $\boldsymbol{\mu}\left(\mathbf{p}_{t}\right)=\boldsymbol{\mu}(\mathbf{p})+\boldsymbol{\delta} \boldsymbol{\mu}$. Substituting these relations in eq. (B.3), we can obtain the bias in our parameter estimation as

$\delta p_{j}=\sum_{i}\left(\mathbf{F}^{-1}\right)_{i j} \operatorname{Tr}\left[\frac{1}{2} \mathbf{C}^{-1} \mathbf{C}_{, i} \mathbf{C}^{-1}\left(\mathbf{C}_{t}-\mathbf{C}\right)+\boldsymbol{\delta} \boldsymbol{\mu}^{T} \mathbf{C}^{-1} \boldsymbol{\mu}_{, i}\right] \simeq \sum_{\mathbf{k}} \frac{1}{\sigma_{k}^{2}} \frac{\partial \mu(\mathbf{k})}{\partial p_{i}} \delta \mu(\mathbf{k}) / \sum_{\mathbf{k}} \frac{1}{\sigma_{k}^{2}} \frac{\partial \mu(\mathbf{k})}{\partial p_{i}} \frac{\partial \mu(\mathbf{k})}{\partial p_{j}}$,

where we again ignored the derivative of the covariance matrix. 